\title{
Evaluation and implementation of highly challenging balance training in clinical practice for people with Parkinson's disease: protocol for the HiBalance effectiveness-implementation trial
}

\author{
Breiffni Leavy ${ }^{1,2^{*}}$ (D, Lydia Kwak ${ }^{3}$, Maria Hagströmer ${ }^{1,4}$ and Erika Franzén ${ }^{1,2,5}$
}

\begin{abstract}
Background: If people with progressive neurological diseases are to avail of evidence-based rehabilitation, programs found effective in randomized controlled trials (RCT's) must firstly be adapted and tested in clinical effectiveness studies as a means of strengthening their evidence base. This paper describes the protocol for an effectiveness-implementation trial that will assess the clinical effectiveness of a highly challenging balance training program (the HiBalance program) for people with mild-moderate Parkinson's disease (PD) while simultaneously collecting data concerning the way in which the program is implemented. The HiBalance program is systemically designed to target balance impairments in PD and has been shown effective at improving balance control and gait in a previous RCT. Study aims are to i) determine the effectiveness of the adapted HiBalance program on performance and self-rated outcomes such as balance control, gait and physical activity level ii) conduct a process evaluation of program implementation at the various clinics iii) determine barriers and facilitators to program implementation in these settings.
\end{abstract}

Methods: This effectiveness-implementation type 1 hybrid study will use a non-randomized controlled design with consecutive inclusion of people with PD at multiple clinical sites. A mixed method approach will be used to collect clinical effectiveness data and process evaluation data which is both quantitative and qualitative in nature. The consolidated framework for implementation research (CFIR) will be used to guide the planning and collection of data concerning implementation barriers and facilitators. The HiBalance program will be provided by physical therapists as a part of standard rehabilitation care at the clinical sites, while the evaluation of the implementation process will be performed by the research group and funded by research grants.

Discussion: An effectiveness-implementation study design benefits patients by speeding up the process of translating findings from research settings to routine health care. Findings from this study will also be highly relevant for those working with neurological rehabilitation when faced with decisions concerning the translation of training programs from efficacy studies to everyday clinical practice.

Trial registration: ClinicalTrials.gov march 2016, NCT02727478.

Keywords: Balance training, Parkinson's disease, Effectiveness-implementation, Pragmatic study design

\footnotetext{
* Correspondence: breiffni.leavy@ki.se

'Department of Neurobiology, Care Sciences and Society, Division of

Physiotherapy, Karolinska Institute, Huddinge, Sweden

${ }^{2}$ Stockholms Sjukhem Foundation, Stockholm, Sweden

Full list of author information is available at the end of the article
} 


\section{Background}

People with Parkinson's disease (PD) experience impairments in balance and gait function, symptoms which can have far-reaching negative effects on their health and quality of life [1-3]. Injurious falls and fear of falling are especially prevalent among those with PD [4, 5], a factor which may partly explain why this group are less physically active than older people of a similar age without the diagnosis [6-8]. Impairments in balance control often present in the early stage of the disease and gradually deteriorate in line with disease progression. Additionally, whereas dopaminergic drugs, the primary treatment method for PD-related motor symptoms, positively affect symptoms such as bradykinesia and tremor, these drugs are also reported to negatively affect components of balance control [9]. Both these factors combined highlight the urgency to develop and implement effective training methods to tackle balance and gait impairments for this patient group.

There is a growing evidence base for the feasibility and effectiveness of balance training in $\mathrm{PD}$ with regards to various aspects of physical function [10-12]. However, previous interventions have been criticized for applying training stimuli which lacked intensity and challenge $[11,13]$. Group-based programs also appear to offer added motivational advantages for people with PD [12, 14], many of whom experience non-motor symptoms such as apathy and depression as a feature of the disease [15]. The HiBalance program [16] was developed with these considerations in mind to specifically address PDspecific balance impairments. This group-based program is highly challenging, progressive and has been proven effective in improving balance and gait impairments in a randomized controlled hospital setting [17]. For an intervention to be considered evidence-based however, it must be proven effective in both research and clinical settings [18].

\section{From randomized trial to routine care}

It is recognized that efficacious interventions frequently require adaptation prior to adoption in clinical settings [19]. This adaptation to clinical factors is often required to ensure feasibility of the intervention the clinical context [20]. Best practice then involves evaluating the effectiveness of the adapted program, in order to verify whether or not the adaptation has attenuated the effective core elements of the program itself [21]. The majority of research trials however do not reach this phase as studies among American and European populations suggest that $30-40 \%$ of patients do not receive care which is in line with the current best scientific evidence [21, 22]. Similarly, in the field of neurological rehabilitation for PD, although there are a growing number of programs tested in randomized controlled environments, the evidence is lacking as to whether the effects of these program can be maintained in real life clinical settings.

Clinical effectiveness research is characterised by the inclusion of more heterogeneous patient samples and settings which, in turn, places greater emphasis on external validity and generalizability of the intervention in question [23, 24]. In this way, effectiveness studies can be considered essential links between scientific evidence and evidence-based practice. There is also empirical evidence to show that the process of implementation is an important determinant of program outcomes [19]. For this reason, evaluating the implementation process provides information regarding the feasibility of intervention programs within real life settings while also providing important contextual information which may help explain the success, or lack thereof, of the tested intervention. When the aim is to maximise the uptake of interventions in primary care, researchers are also advised to examine the influence of contextual factors on implementation, as this will give an indication of the 'fit' between the intervention and the context within which it is being embedded [25].

\section{Rationale for trial design}

The effectiveness-implementation hybrid design [26] enables the simultaneous evaluation of both effectiveness outcome measures as well as of the process of implementation. This design is therefore hypothesized to speed up the implementation process of interventions within healthcare [26]. The type 1 Hybrid design is suitable when a study aims to both test effectiveness of a clinical intervention (outcome evaluation) while simultaneously gathering contextual data regarding potential barriers and facilitators to clinical implementation. Use of the hybrid design allows for constant monitoring of the process by which the intervention is applied, and therefore allow problems in early application to be identified and quickly altered so as to ensure better outcomes [19]. Non-randomization in the current study is a design trade-off which was made in order to ensure that implementation of the program is practically feasible at the various clinical sites within a reasonable time-frame. Lastly, we will adhere to a participatory approach whereby 'users' of the program (physical therapist trainers) will be actively involved in all stages of the program adaptation, process and outcome evaluation. This approach is recommended in order to increase the relevance, acceptability and successful implementation of the program [21].

\section{Study aims}

This study has three main aims which involve testing the effectiveness of the HiBalance program: 
Aim I: to test the effectiveness of the adapted balance training program on balance, gait and physical activity in people with PD in real-life clinical settings.

Aim II: to conduct a process evaluation of the effectiveness-implementation study by gathering information on the implementation process at the various clinical settings.

Aim III: to determine barriers and facilitators that affect implementation of the program in these settings.

The study results will test the hypothesis that the effectiveness of the HiBalance program can be retained following adaptation of the intervention and study results should inform future translation of evidence-based training programs in clinical settings among people with PD.

\section{Methods}

\section{Description of the HiBalance program}

The intervention involves a 10-week highly challenging and progressive balance training program in group format and has been previously described [16]. The grouptraining exercises are not fixed but selected to target four major components of balance control which are known to be impaired among people with PD; (i) Sensory integration; (ii) Anticipatory postural adjustments; (iii) Motor agility and (iv) Stability limits. Trainers are responsible for the planning, selection and adaption of the exercises as well as ensuring that exercises are adapted to participants' individual capacity. Successive progression of exercise difficulty is achieved by dividing the 10 -week period into three blocks A (2 weeks), B (4 weeks) and C (4 weeks). During block $\mathrm{A}$, the focus involves learning the exercises and ensuring quality of the performance. During block B, the level of difficulty of the exercises increases and cognitive as well as motoric dual task exercises are introduced, 1 week at a time. During block $\mathrm{C}$, exercises from all four balance components are combined in order to increase the complexity of training and participants are required to switch between cognitive and motoric dual task exercises during the same training session.

\section{Adaptation of the HiBalance program to clinical settings} Adaptation of the HiBalance program was a measure taken in the preparation phase to ensure its applicability in the clinical context. An overview of these adaptations is outlined in Table 1. The adaptation process occurred during a series of peer-group meetings between the research team, clinical trainers who had previously participated in the RCT as well as future clinical trainers. Reducing the dose of the group training sessions from 30 to 20 hours was motivated primarily by current regulations for rehabilitation funding in the Swedish national healthcare system. Development and adaptation of the home training program was based on trainer and participant perceptions of the exercises, gathered during the pilot study using questionnaires (participants) and focus group interviews (trainers). Lab-based and time consuming outcomes assessments employed during the efficacy trial were substituted with more clinically feasible assessments. A pilot study (data not published) was then performed at two clinic settings in order to test the feasibility of these adaptations. The objective of the pilot study was to test the main uncertainties identified in the development work prior to the effectivenessimplementation study [21].

All physical therapist trainers will participate in $2 \mathrm{x}$ $3 \mathrm{~h}$ sessions where they will receive theoretical and practical training in the HiBalance program as well as information regarding the project design and goals. Project materials will be provided covering all aspects of recruitment, training and measurement and regular support will be achieved with the research team though contact by mail, telephone and meetings.

The following section outlines the study setting, and for each study aim, participants, data collection procedures, and analysis plans are described.

\section{Study setting}

The study will be conducted in 4-6 rehabilitation clinics ranging in nature from primary care geriatric clinics to out-patient clinics specializing in neurological physiotherapy rehabilitation in Stockholm, Sweden. In Sweden patients can receive out-patient physiotherapy treatment without doctor/specialist referral and number of treatments is decided upon on an individual basis. Health care treatment cost is tax-funded and clinics receive funding reimbursements for an average of eight treatment sessions/patient during 1 year. Cost to the patient is also regulated whereby in a 1 year period, individual sessions cost an equivalent of 23 US dollars up to ceiling of approx. 120 dollars, from which point onwards rehabilitation treatment is free of charge during the remaining year.

\section{Study phases}

When describing the varying phases of this study (Fig. 1) we use taxonomy from Aaron's conceptual model for the dynamic adaptation process [27]. According to this model, the implementation process can be divided into four phases; exploration; adoption/preparation; implementation and sustainment. In Fig. 1 we describe the first three of these stages of Aaron's model and label the third or 'implementation' stage as the 'Outcome/Process Evaluation' as it applies to the current study. We deem that the current study will be followed by post evaluation scale-up where implementation will be tested on a wider scale, before the sustainment stage of the HiBalance program is actual. In the preparation phase for this 
Table 1 HiBalance program design differences between the efficacy and effectiveness-implementation stages

\begin{tabular}{|c|c|c|}
\hline Program feature & HiBalance-RCT (Efficacy phase) & HiBalance-clinical setting (Effectiveness/Implementation phase) \\
\hline \multirow[t]{5}{*}{ Inclusion criteria } & Idiopathic Parkinson's disease & Idiopathic Parkinson's disease \\
\hline & Hoehn \& Yahr score of 2 or 3 & Hoehn \& Yahr score of 2 or 3 \\
\hline & Able to walk independently indoors without an aid & Able to walk independently indoors without an aid \\
\hline & Mini-Mental State examination score $>24$ points & Cognitively capable of following instructions in a group setting \\
\hline & Age $\geq 60$ years & All Ages \\
\hline $\begin{array}{l}\text { Core } \\
\text { components }\end{array}$ & $\begin{array}{l}\text { Individually adapted, highly challenging and progressive } \\
\text { balance training in } 3 \text { blocks with progressively integrated } \\
\text { dual-task training }\end{array}$ & $\begin{array}{l}\text { Individually adapted, highly challenging and progressive balance } \\
\text { training in } 3 \text { blocks with progressively integrated dual- } \\
\text { task training }\end{array}$ \\
\hline Dose & $\begin{array}{l}30 \mathrm{~h} \text { of group training } \\
(3 \times 1 \mathrm{~h} \text { sessions/week } \times 10 \text { weeks })\end{array}$ & $\begin{array}{l}20 \mathrm{~h} \text { of group training }(2 \times 1 \mathrm{~h} \text { sessions/week } \times 10 \text { weeks }) 10 \mathrm{~h} \\
\text { home exercise program }(1 \mathrm{~h} / \text { week } \times 10 \text { weeks })\end{array}$ \\
\hline Providers & $\begin{array}{l}\text { Physical therapist PhD students (site responsible) and } \\
\text { clinicians }\end{array}$ & Physical therapist clinicians \\
\hline Sites & 2 sites, one university hospital & 4-6 clinical sites/primary care clinics \\
\hline \multicolumn{3}{|l|}{ Outcome evaluation } \\
\hline \multicolumn{3}{|l|}{ Performance-based } \\
\hline \multirow{2}{*}{$\begin{array}{l}\text { Balance } \\
\text { performance }\end{array}$} & Mini-BESTest score & Mini-BESTest score \\
\hline & Modified figure of eight test & \\
\hline $\begin{array}{l}\text { Physical activity } \\
\text { level }\end{array}$ & Steps per day measured by accelerometer & Steps per day measured by accelerometer \\
\hline \multicolumn{3}{|l|}{ Self-reported } \\
\hline $\begin{array}{l}\text { Fear of falling/ } \\
\text { balance confidence }\end{array}$ & $\begin{array}{l}\text { Falls Efficacy Scale-International } \\
\text { (FES-I) } \\
\text { (A measure of concerns about falling) }\end{array}$ & $\begin{array}{l}\text { Activities-specific balance confidence scale (ABC scale) } \\
\text { (A measure of balance confidence) }\end{array}$ \\
\hline $\begin{array}{l}\text { Activities of daily } \\
\text { living }\end{array}$ & $\begin{array}{l}\text { Unified Parkinson's Disease Rating Scale (UPDRS)- ADL } \\
\text { component }\end{array}$ & \\
\hline $\begin{array}{l}\text { Self-rated health } \\
\text { Walking } \\
\text { Evaluation method }\end{array}$ & $\begin{array}{l}\text { SF-36/PDQ-39 } \\
\text { PDQ-39 } \\
\text { Randomized controlled trial }\end{array}$ & $\begin{array}{l}\text { EQ-5D-3 L } \\
\text { Walking impact scale (Walk 12G) } \\
\text { Non-randomized controlled design }\end{array}$ \\
\hline
\end{tabular}

study (Fig. 1) we will also, using a web-questionnaire, assess the needs and resources of registered physical therapists in Sweden in relation to their work with people with PD. The web questionnaire will be sent to all physical therapists registered in the neurology, geriatrics and out-patient care sections of the national organization in Sweden. This information will then be used at the later stage to inform wider-scale implementation.

\section{Outcome evaluation of the intervention (Aim I)}

The primary and secondary outcome measures of the intervention will be assessed using a non-randomized controlled clinical trial design. Eligible participants will be consecutively included to the training groups, which mirrors the standard approach in physical therapy rehabilitation, and participants will be recruited both internally from within the clinics and in response to advertisement in local newspapers.

\section{Study participants}

Inclusion criteria for the trial are outlined in Table 1. A broadening of the age criteria in the current trial will enable community-dwelling people at mild-moderate stages of PD of all ages to be eligible for inclusion. Power analyses will determine sample-size using data from the Pilot study performed in the fall of 2015 as well as the previous HiBalance RCT. Control participants will be recruited and tested in a similar manner as those involved in the intervention group. Control subjects will be encouraged to continue their usual daily activities during the 10-week period between measurements and will not be advised against participating in other training interventions.

\section{Data collection}

The testing procedure is also designed to reflect that which occurs in real-life clinical practice and participants will be tested by physical therapists at baseline (pre-training) and at 10-weeks (post training). Data collection will occur at the respective clinics and be comprised of both clinical performance tests as well at selfreported questionnaires. All participants will be tested during the on-phase of their medication and testing will 


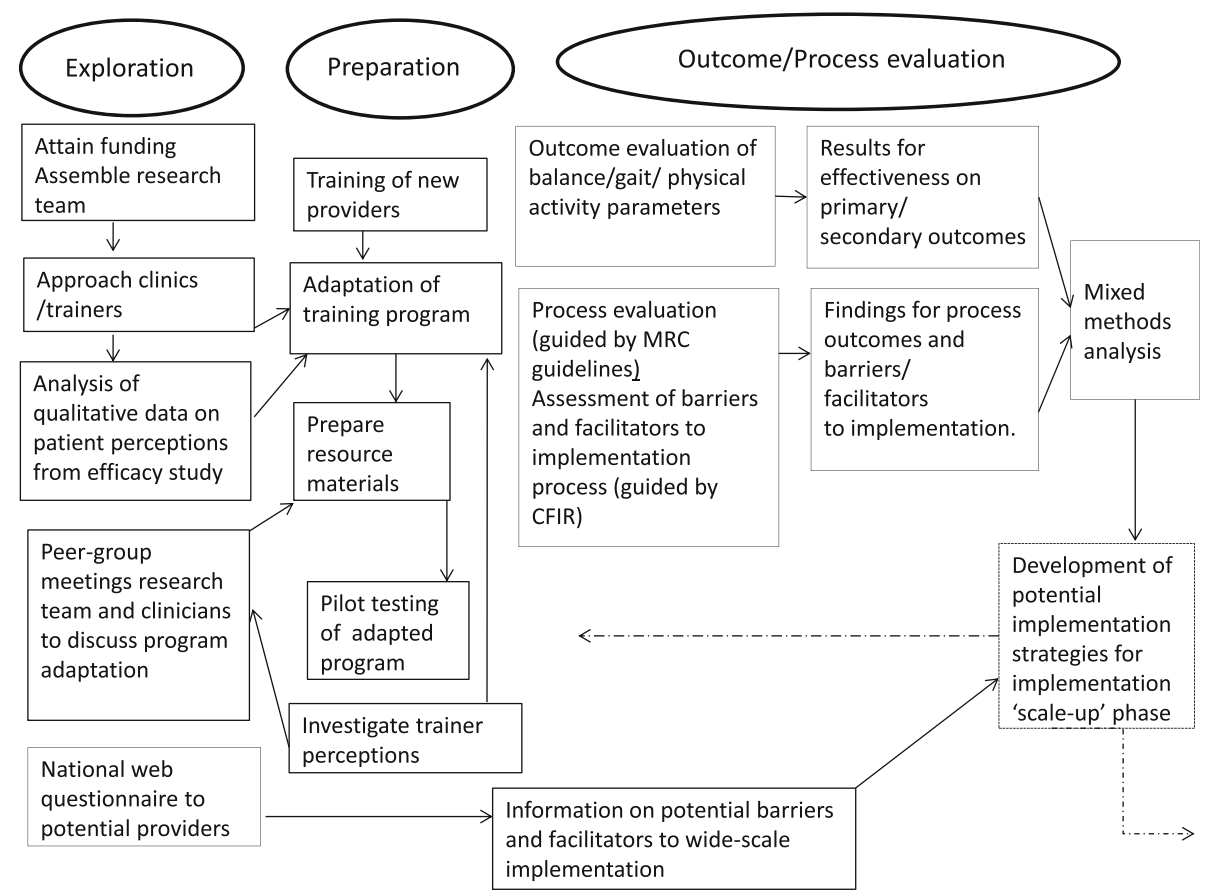

Fig. 1 Overview of study phases for the HiBalance effectiveness-implementation trial

be scheduled to occur at the same time on both test occasions.

\section{Outcome measures}

Both performance-based and self-reported outcome measures will assess the effectiveness of the intervention (Table 1). The primary outcome measure is balance performance which will be assessed using the 14-item Mini Balance Evaluation Systems test (Mini-BESTest) [28]. This test assesses four components of balance control; anticipatory postural adjustments, postural responses, sensory orientation, and stability in gait which are directly targeted in the HiBalance program. Secondary outcomes will include gait velocity (measured by the 10-meter Walking Test); functional mobility (measured by Timed Up and Go test [29]); physical activity level (measured as steps per day using a waist-worn accelerometer (Actigraph GT3X+, Pensacola, FL, USA). In addition to performance-based outcome measures, patient-reported outcomes will be used. Balance confidence will be measured using the Swedish version of the Activities-specific balance confidence $(A B C)$ scale [30]. This scale requires respondents to rate their confidence to maintain balance during 16 different real-life situations. The ABC scale substitutes the FES (I) scale used in previous evaluations of HiBalance [17] as this scale poses questions concerning a wider range of potentially difficult and outdoor activities and is considered more suitable to detect changes in moderate to highly functioning adults [31]. Self-rated health will be measured using the EurQol's EQ- 5D [32]. The EQ-5D$3 \mathrm{~L}$ is a standardized non-disease-specific instrument for describing health-related quality of life, and is a measure already in use within rehabilitation in Sweden. When using this instrument, respondents are required to rate their health status in relation to 5 dimensions; mobility, self-care, usual activities, pain/discomfort and anxiety/depression. Subjective walking ability will be assessed using the Swedish version of the Walking impact scale (walk 12G) [33], a 12-item generic patient-reported rating scale measuring walking difficulty in everyday life as experienced during the previous 2 weeks.

\section{Data analysis}

Sample size calculation was based on the results from the pilot study (unpublished data) and calculated in relation to the primary outcome of balance control. In order to attain $80 \%$ power with a two-sided alpha level of $5 \%$, the number of subjects required per group and the hypothesized effect size (of 1 point according to the MiniBESTest) was 37 . With an expected drop out rate of $15 \%$ during the course of the trial, this calculation will require the inclusion of approximately 45 participants in each group. The distribution of variables which can be thought to confound study outcomes will be compared at baseline between the control and intervention groups using the Student $t$ test, Mann-Whitney or Chi-squared test, where appropriate. Where differences between groups are found we plan to adjust for covariates which 
are known to affect outcomes in PD. Where data is seen to be of skewed distribution, logarithmic transformations or non-parametric statistics will be used to assess the effectiveness of the intervention. Repeated measures analysis of variance test or mixed models, where appropriate, will test for interaction effects between the intervention and control groups, post-hoc tests will be used when significant interactions are found. All analysis will be performed using the intention-to-treat principle and 95\% confidence intervals will be calculated throughout.

\section{Process evaluation of the implementation (Aim II)}

In order to make an accurate interpretation of the outcomes outlined above it is essential to understand the quality and quantity of the intervention that was actually delivered. For this reason, we will conduct a process evaluation of the implementation of the effectiveness study.

\section{Research question relating to aim II}

To what extent was the HiBalance program delivered as intended and how was the delivery conducted?

The process evaluation will be guided by the UK medical research council's recommendations for planning and conducting process evaluation of complex interventions [21]. Process evaluations involve critically observing the work of the clinicians who are providing the training intervention [21]. Participants involved in this part of the study will therefore also include physical therapist trainers at the respective clinical sites. Due to the small numbers in individual clinics we will lack the power to statistically assess site-based factors that influence implementation outcomes. We will therefore use a mixed methods approach when evaluating the implementation process. This will be performed by data collection from trainers, patients and professionals in the field using interviews, questionnaires, analysis of patient training diaries as well as physical therapist planning protocols. We will, in accordance with the current implementation literature, adopt measures to support the implementation process by; adapting the HiBalance program to the clinical context; engaging in shared-decision making with project clinician trainers; increasing trainer skills and by providing ongoing administrative and technical support [19].

\section{Assessment of process outcomes}

In order to fulfill the aims of the process evaluation which are to examine the quality and quantity of what was delivered it is necessary to assess outcomes such as the fidelity and dose of the program as it was delivered, as well as describe the recruitment process and reach of the intervention (Table 2) [21].
Fidelity is defined as the degree to which the intervention is implemented as prescribed or intended by the program developers [34]. We aim to perform a fidelity assessment of the implementation process by monitoring the delivery of the core components of the HiBalance program, as it was designed. Adherence to these core components as well as the week-by-week structure of the sessions will be quantitatively assessed through collection and analysis of the planning materials used by trainers to plan the group training sessions (Additional file 1). Trainers at each clinic will be advised to dedicate approximately $20 \mathrm{~min}$ to planning training sessions, inspired by program materials, and to document training protocols following each session. Program features such as the warm-up phase of the training sessions, on the other hand, can be altered to achieve a better fit to specific clinics [19]. Fidelity will also be assessed qualitatively using open ended questions during focus-group interviews where trainers will be allowed describe this feature in their own words.

Dose: Both the dose of group training sessions delivered at the clinical sites, and the dose received by patients will be assessed. Dose received involves rate of participation in both group and home training sessions by participants. This data will be quantitatively assessed using attendance protocol as well as home training diaries.

Recruitment: the process of recruitment will be described quantitatively for the various clinics and trainers perceptions of recruitment will be qualitatively explored during the focus group interviews.

Reach refers to the extent to which the target audience comes into contact with the intervention [20]. We plan to describe to which extent those included in the study are representative of the target population of people with PD.

Assessing barriers and facilitators to implementation (Aim III) In addition to evaluating 'how' and 'what' was implemented by means of the process evaluation we will collect data concerning potential barriers and facilitators to the implementing the HiBalance program.

\section{Research question relating to aim III}

What are the potential barriers and facilitators to implementation of the HiBalance training program in outpatient clinical settings?

\section{Determinant framework}

The Consolidated Framework for Implementation Research (CFIR) will be used in the current study to guide the investigation of potential barriers and facilitators of the implementation process [35]. CFIR can be categorized as a determinant framework, in that it can be used 
Table 2 Overview of the process evaluation involving process outcomes and barriers and facilitators, targeted groups and data collection methods

\begin{tabular}{|c|c|c|}
\hline Process outcomes $^{\mathrm{a}}$ & Targeted groups & Method of data collection \\
\hline Fidelity & $\begin{array}{l}\text { Patient training diaries/ } \\
\text { attendance } \\
\text { Physical therapist trainers sessions plans }\end{array}$ & $\begin{array}{l}\text { Assessment of group training } \\
\text { protocols } \\
\text { Focus group interviews }\end{array}$ \\
\hline Dose & $\begin{array}{l}\text { Physical therapist trainers (dose } \\
\text { provided) } \\
\text { Participants with PD } \\
\text { (dose received) }\end{array}$ & $\begin{array}{l}\text { Assessment of group training } \\
\text { protocols } \\
\text { Assessment of attendance at } \\
\text { group training/home training } \\
\text { protocols }\end{array}$ \\
\hline Recruitment & Physical therapist trainers & $\begin{array}{l}\text { Study logs and focus group } \\
\text { interviews }\end{array}$ \\
\hline Reach & Participants with PD & Study logs \\
\hline Barriers and facilitators ${ }^{b}$ & Targeted groups & Method of data collection \\
\hline $\begin{array}{l}\text { Knowledge and beliefs about the program } \\
\text { Relative advantage of the program } \\
\text { Need for adaptability of the program } \\
\text { Self-efficacy to train patients according to the programs core } \\
\text { components } \\
\text { Perceived complexity/difficulty of implementing the program in everyday } \\
\text { practice }\end{array}$ & Physical therapist trainers & Focus group interviews \\
\hline $\begin{array}{l}\text { Patients need and resources } \\
\text { Knowledge and beliefs about the balance program } \\
\text { Perceived complexity of performing the training }\end{array}$ & Participants with PD & Structured questionnaire \\
\hline
\end{tabular}

to identify determinants (i.e. barriers and facilitators) at different levels (from the user to the program provider, to the organizational level) that can be thought to influence the implementation process [36]. CFIR provides a menu of constructs which are operationally defined and stem from various disciplines such as psychology and sociology [35]. We will choose constructs which we hypothesize to specifically impact the implementation of the HiBalance program from the following main domains defined by CFIR; Intervention characteristics; Inner setting; Outer setting and Characteristics of individuals. Table 2 provides an overview of the process outcomes as well as the subdomains of the CFIR constructs outlines above, which will guide data collection of the process evaluation and in the assessment of barriers and facilitators to implementation. However, we also acknowledge such frameworks do not conceptualize the role of unexpected events [37] and we aim therefore for flexibility in the data collection to allow for emergent factors concerning barriers and facilitators to be explored during the qualitative interview process [21].

\section{Data collection}

Data concerning aims II and III of the study will be collected using a mixed method approach. Focus group interviews will be performed with 3-6 physical therapists at the various clinical sites as a means to explore the process outcomes/barriers and facilitators outlined in Table 2. We will follow guidelines published by CFIR when developing the interview guide (http:// cfirguide.org/tools.html). Additional file 2 demonstrates how interview questions are linked to CFIR constructs. Interviews will aim to gather detailed information regarding trainer experiences and perceptions throughout the evaluation period, in both the early and late stages of the process. Additionally, structured questionnaires will be used to capture aspects of patient experiences of the program upon completion of the 10 -week training (Additional file 3).

\section{Data analysis}

Interviews transcripts will be systematically analyzed using thematic qualitative content analysis. This method is systematic, replicable and valid method in the analysis of text data [38]. During data analysis the research team will strive for an inductive approach to category development, by allowing categories to emerge from the data as opposed to driving category development by preconceived theory. The analysis will be performed systematically in steps [39], involved research group debriefing sessions which allow for researcher triangulation and ensure validity of the analysis process. Questionnaires and planning protocols will be analyzed primarily using descriptive statistics.

\section{Discussion}

If evidence-based training programs are to benefit those with progressive neurological diseases, such as PD, the findings from RCT's must be translated to and tested in everyday clinical settings. Additionally, there are a 
scarcity of studies which clearly report the underlying principles upon which such exercise programs are based [40]. The HiBalance program described in the current protocol has, on the other hand, been based on specific underlying theoretical principles and been shown effective in an RCT [17]. For these reasons this program is suitable for testing in an effectiveness study with the aim of further implementation on a wider scale. We find to date, no previously published trials which have tested the effectiveness of training interventions in PD while simultaneously evaluating the way in which the program was implemented.

By using a hybrid design we intend to fill gaps in the current knowledge concerning not only what to implement but also how to implement evidence-based balance training for mild to moderate PD in clinical practice. This study will therefore determine whether the adapted HiBalance program is effective at improving balance control, gait and physical activity level when provided in standard physiotherapy practice. Our results will also indicate whether implementation of the program is feasible on a wider scale in the Swedish context and what potential barriers and facilitators to this process may be. In this study protocol we use a theoretical approach to outline the core elements of the implementation process. We also hypothesize that results from our study will be highly relevant for those working within the field of neurological rehabilitation when considering ways in which to translate research findings into routine clinical care. Use of predefined constructs regarding the planned process evaluation also strengthens our design by enabling the easy comparison of our results with those of other studies concerning the implementation of healthcare interventions.

There will also be possible limitations to the proposed study. Firstly, this intervention does not consist of a fixed program of balance exercises, but relies on trainers' adherence to the basic exercise principles in order to plan and adapt training sessions. While this feature allows exercises to be adapted and progressed at the individual level, it may be difficult to fully evaluate program fidelity. Extra efforts will therefore need to be made to evaluate the level of fidelity to the theoretical principles of the program throughout the implementation process. Secondly, randomization of participants into treatment and control groups was not considered feasible as the program is provided at the clinical sites as a part of standard physiotherapy treatment. Although consecutive inclusion of participants mirrors standard practice and therefore benefits external validity, a non-randomized design limits our ability to rule out the effects of selection bias between the control and intervention groups. Thirdly, in current physiotherapy treatment of people with PD in Sweden, there is no uniform standard care with which to compare the testing of this intervention. This may hamper the extent to which a standardized control group can be recruited.

This study represents a unique and structured approach to investigating the ways in which a rehabilitation intervention with proven efficacy responds to testing and implementation in real life clinical settings. Our study design should benefit people with progressive neurological conditions such as PD by providing valuable information which can speed up the process of translating research findings to routine health care. We hypothesize that study findings will produce highly applicable results not only for those in the field of PD, but to clinicians on a wider scale who are planning to translate research findings from training programs with proven effectiveness to the patients who need them.

\section{Trial status}

At the time of manuscript submission the trial is in an ongoing phase of recruitment and data collection at the various clinical sites.

\section{Additional files}

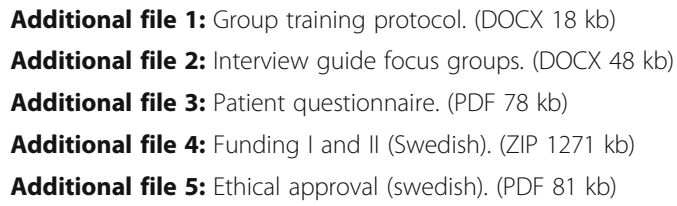

\section{Abbreviations}

CFIR: Consolidated framework for implementation research; PD: Parkinson's disease; RCT: Randomized controlled trial

\section{Acknowledgements}

The authors wish to acknowledge all physical therapist trainers actively involved in the adaptation, provision and assessment of this phase of the HiBalance trial at the various clinical sites in Stockholm (Karolinska University Hospital; The Stockholm Sjukhem Foundation at their rehab centers on Kungsholmen and Bromma: The Stora Sköndal Foundation and Nacka rehab center) without whose effort this study would not be possible.

\section{Funding}

This study has received research grant funding after competitive peer-review from the following sources: The Swedish Research council for Health, Working life and Welfare (FORTE), The Swedish Research Council, Vårdalstifelsen' and the Swedish Parkinson Foundation (Additional files 4i-ii). The funding agencies did not influence study design and will not play an influential role in data analysis or interpretation.

\section{Availability of data and materials}

The datasets generated and analyzed during the current study will be available from the author upon reasonable request.

\section{Authors' contributions}

EF is principal investigator and conceived the study along with $\mathrm{MH}$ and LK, as well as acquired all study funding. All authors participated in development of study design. BL and EF designed the adaptation of the program and will provide support to trainers throughout. LK and BL designed the process evaluation part of the study encompassing aim II and III. All authors read, reviewed and approved the final manuscript. 


\section{Competing interests}

The authors declare that they have no competing interests.

\section{Consent for publication}

Not applicable.

\section{Ethics approval and consent to participate}

All participants, both people with PD and physical therapist trainers wil provide their written consent for participation in the study. This study has been approved by the Regional Ethical Review Board in Stockholm (2016/201-31/12) (Additional file 5).

\section{Author details}

'Department of Neurobiology, Care Sciences and Society, Division of Physiotherapy, Karolinska Institute, Huddinge, Sweden. ${ }^{2}$ Stockholms Sjukhem Foundation, Stockholm, Sweden. ${ }^{3}$ Institute of Environmental Medicine, Intervention and implementation research, Karolinska Institute, Solna, Sweden. ${ }^{4}$ Function Area Occupational therapy \& Physiotherapy, Allied Health Professionals Function, Karolinska University Hospital, Stockholm, Sweden. ${ }^{5}$ Allied Health Professionals Function, Karolinska University Hospital, Stockholm, Sweden.

\section{Received: 11 November 2016 Accepted: 26 January 2017} Published online: 07 February 2017

\section{References}

1. Rahman S, et al. Quality of life in Parkinson's disease: the relative importance of the symptoms. Mov Disord. 2008;23(10):1428-34.

2. Kerr GK, et al. Predictors of future falls in Parkinson disease. Neurology. 2010; 75(2):116-24.

3. Paul SS, et al. The relative contribution of physical and cognitive fall risk factors in people with Parkinson's disease: a large prospective cohort study. Neurorehabil Neural Repair. 2014;28(3):282-90.

4. Parashos SA, et al. Falls in Parkinson disease: analysis of a large crosssectional cohort. J Parkinsons Dis. 2013;3(4):515-22.

5. Pickering RM, et al. A meta-analysis of six prospective studies of falling in Parkinson's disease. Mov Disord. 2007:22(13):1892-900.

6. Fertl E, Doppelbauer A, Auff E. Physical activity and sports in patients suffering from Parkinson's disease in comparison with healthy seniors. J Neural Transm Park Dis Dement Sect. 1993;5(2):157-61.

7. van Nimwegen M, et al. Physical inactivity in Parkinson's disease. J Neurol. 2011;258(12):2214-21.

8. Benka Wallen $\mathrm{M}$, et al. Levels and patterns of physical activity and sedentary behavior in elderly people with mild to moderate Parkinson disease. Phys Ther. 2015;95(8):1135-41.

9. Hall LM, et al. The effect of Parkinson's disease and levodopa on adaptation of anticipatory postural adjustments. Neuroscience. 2013;250:483-92.

10. Tomlinson $\mathrm{CL}$, et al. Physiotherapy versus placebo or no intervention in Parkinson's disease. Cochrane Database Syst Rev. 2013;9:CD002817.

11. Allen NE, et al. Balance and falls in Parkinson's disease: a meta-analysis of the effect of exercise and motor training. Mov Disord. 2011;26(9):1605-15.

12. Shen $X$, Wong-Yu IS, Mak MK. Effects of exercise on falls, balance, and gait ability in Parkinson's disease: A meta-analysis. Neurorehabil Neural Repair. 2016;30(6):512-27.

13. Abbruzzese G, Marchese R, Avanzino L, Pelosin E. Rehabilitation for Parkinson's disease: Current outlook and future challenges. Parkinsonism Relat Disord. 2016;22 Suppl 1:S60-4.

14. King LA, et al. Effects of group, individual, and home exercise in persons with Parkinson disease: a randomized clinical trial. J Neurol Phys Ther. 2015; 39(4):204-12.

15. Chaudhuri KR, Schapira AH. Non-motor symptoms of Parkinson's disease: dopaminergic pathophysiology and treatment. Lancet Neurol. 2009;8(5): 464-74.

16. Conradsson D, et al. A novel conceptual framework for balance training in Parkinson's disease-study protocol for a randomised controlled trial. BMC Neurol. 2012;12:111.

17. Conradsson $\mathrm{D}$, et al. The effects of highly challenging balance training in elderly with Parkinson's disease: a randomized controlled trial. Neurorehabil Neural Repair. 2015;29(9):827-36.

18. Fraser $M$, et al. Intervention research- developing social programs. New, York: Oxford University Press; 2009.
19. Durlak JA, DuPre EP. Implementation matters: a review of research on the influence of implementation on program outcomes and the factors affecting implementation. Am J Community Psychol. 2008:41(3-4):327-50.

20. Brownson RC, Colditz G, Proctor E. Dissemination and implementation research in health- translating science to practice. New York: Oxford University Press; 2012.

21. Moore GF, et al. Process evaluation of complex interventions: medical research council guidance. BMJ. 2015;350:h1258.

22. Grol R, Grimshaw J. From best evidence to best practice: effective implementation of change in patients' care. Lancet. 2003;362(9391):1225-30.

23. Bauer MS, et al. Principles of effectiveness trials and their implementation in VA cooperative study \#430: 'reducing the efficacy-effectiveness gap in bipolar disorder'. J Affect Disord. 2001;67(1-3):61-78.

24. Wells KB. Treatment research at the crossroads: the scientific interface of clinical trials and effectiveness research. Am J Psychiatry. 1999;156(1):5-10.

25. Lau $R$, et al. Achieving change in primary care-causes of the evidence to practice gap: systematic reviews of reviews. Implement Sci. 2016;11(1):40.

26. Curran GM, et al. Effectiveness-implementation hybrid designs: combining elements of clinical effectiveness and implementation research to enhance public health impact. Med Care. 2012;50(3):217-26.

27. Aarons GA, Hurlburt M, Horwitz SM. Advancing a conceptual model of evidence-based practice implementation in public service sectors. Adm Policy Ment Health. 2011;38(1):4-23.

28. Franchignoni $F$, et al. Using psychometric techniques to improve the balance evaluation systems test: the mini-BESTest. J Rehabil Med. 2010;42(4): 323-31.

29. Podsiadlo D, Richardson S. The timed "Up \& Go": a test of basic functional mobility for frail elderly persons. J Am Geriatr Soc. 1991;39(2):142-8.

30. Powell LE, Myers AM. The activities-specific balance confidence (ABC) scale. J Gerontol A Biol Sci Med Sci. 1995;50A(1):M28-34.

31. Myers AM, et al. Discriminative and evaluative properties of the activitiesspecific balance confidence (ABC) scale. J Gerontol A Biol Sci Med Sci. 1998; 53(4):M287-94.

32. Brooks R. EuroQol: the current state of play. Health Policy. 1996;37(1):53-72.

33. Bladh $\mathrm{S}$, et al. Psychometric performance of a generic walking scale (Walk-12G) in multiple sclerosis and Parkinson's disease. J Neurol. 2012;259(4):729-38.

34. Dusenbury $L$, et al. A review of research on fidelity of implementation: implications for drug abuse prevention in school settings. Health Educ Res. 2003;18(2):237-56.

35. Damschroder $L$, et al. Fostering implementation of health services research findings into practice: a consolidated framework for advancing implementation science. Implement Sci. 2009;4:50.

36. Nilsen P. Making sense of implementation theories, models and frameworks Implement Sci. 2015;10:53.

37. McAlearney AS, et al. Challenges of implementation and implementation research: Learning from an intervention study designed to improve tumor registry reporting. SAGE Open Med. 2016;4:2050312116666215.

38. Krippendorff K. Content analysis: an introduction to its methodology. Beverly Hills: Sage; 1980.

39. Graneheim UH, Lundman B. Qualitative content analysis in nursing research: concepts, procedures and measures to achieve trustworthiness. Nurse Educ Today. 2004;24(2):105-12.

40. Goodwin VA, et al. The effectiveness of exercise interventions for people with Parkinson's disease: a systematic review and meta-analysis. Mov Disord. 2008:23(5):631-40. 